\title{
A Prescription for misunderstanding: Opportunities for misinterpretation along the information flow from physician to patient
}

\author{
Kevin R. Harris ${ }^{1}$, Gary L. Bradshaw ${ }^{2}$, Karen Koch $^{3}$, James Whyte I V \\ 1. Department of Psychology, Austin Peay State University, Clarksville, the United States. 2. Department of Psychology, \\ Mississippi State University, Mississippi, the United States. 3. North Mississippi Medical Center, Mississippi, the United \\ States. 4. College of Nursing, Florida State University, Tallahassee, the United States.
}

Correspondence: Kevin R. Harris. Address: Department of Psychology, Austin Peay State University, Clarksville, the United States. Email: harrisk@apsu.edu

Received: May 1, 2013

DOI : $10.5430 /$ jnep.v4n5p1
Accepted: November 25, 2013 Online Published: February 18, 2014

URL: http://dx.doi.org/10.5430/jnep.v4n5p1

\section{Abstract}

Background: Medication errors are costly in both human and financial loss. Interpretations of prescription instructions have been found to vary considerably, including discrepancies between patients and prescribing physicians.

Objective: Identify discrepancies in interpretation of prescription instructions between healthcare consumers, nurses, and physicians

Method: Research Design: Cross-sectional study; Setting: Large university, 2 hospitals and various clinics in Mississippi and Florida; Participants: 74 young healthcare consumers, 34 RNs, and 36 physicians; Measures: Questionnaires asking for interpretations (i.e., what times would/should you take the drug) for various prescription instructions were provided to healthcare consumers, nurses, and physicians.

Results: There was considerable within-group variability in the interpretation of prescription instructions by all groups including physicians. Moreover, physicians, nurses, and healthcare consumers exhibited between group variability in their interpretation of prescription instructions. None of the instructions were uniformly interpreted and a fair number of consumer and nurse interpretations resulted in potentially unsafe schedules of drug administration. Some physicians and nurses also apparently lacked awareness of the potential for interpretation variability.

Conclusions: Because two healthcare providers can have different intentions for identical instructions, an awareness and subsequent education of potential sources of misinterpretation is vital. The present results indicate a need to identify and explore within-group variability of intent among physicians and other healthcare providers.

\section{Key words}

Prescription, Medication, Variability, Instruction, Physician intent

\section{I ntroduction}

Medical errors in the United States annually cost an estimated $\$ 17$ to $\$ 29$ billion and 44,000 to 98,000 lives ${ }^{[1]}$. Medicationrelated errors account for $19.4 \%$ of injuries associated with medical errors, resulting an estimated 34,920 annual deaths in 
hospitals ${ }^{[2]}$ and a reported 1,459 outpatient deaths in a single year ${ }^{[3]}$. Yet many people do not adhere to a drug regimen as prescribed by their physician. The World Health Organization (WHO) released a report intended to call attention to low rates of adherence globally ${ }^{[4]}$. The WHO's report included a brief review of studies addressing patient adherence to medications prescribed over the long term and found adherence rates as low as $27 \%$. Although the adherence rate varied based upon factors such as the type of drug or country under investigation, the overall conclusion was that adherence rates over the long-term was about $50 \%$ in developed countries. Given the grave consequences and the apparently low rate of adherence, the question remains as to what can be done to improve adherence.

Because adherence depends critically on the patient's correct interpretation of the intention of the prescribing physician, misunderstanding of prescription instructions has been the focus of numerous reports. In an early example, $51 \%$ of outpatients at a Rochester, New York hospital did not adhere to their prescriptions and half of the non-adherent patients indicated that they did not understand the instructions ${ }^{[5]}$. Similarly, among home-care patients, 36 out of 40 (90\%) patients made at least one error in adhering to the prescription instructions ${ }^{[6]}$. One investigation found that all ten prescriptions tested were misunderstood by at least one patient ${ }^{[5]}$. Recently, researchers have identified limited reading ability as a strong indicator of risk that a patient will misinterpret prescription instructions ${ }^{[7,8]}$. For example, Wolf et al. presented patients with five dosage instructions and found that the instructions were misunderstood at rates ranging from $8 \%$ to $33 \%$. Moreover, patients exhibiting low literacy misunderstood the instructions $63 \%$ of the time (patients with adequate literacy still misunderstood $38 \%$ of the time) ${ }^{[8]}$.

We propose that the flow of information required in order for a prescribed drug to reach the hands of a patient provides multiple opportunities for misinterpretation to occur - from physician, pharmacist, nurse/caretaker, and ending with the patient (see Figure 1). A physician or other healthcare provider may prescribe a specific drug or multiple drugs following diagnosis of the patient's illness. The prescription order is then processed by a pharmacist and provided directly to the patient or to other healthcare providers, whom are responsible for providing the drug(s) to the patient. These multiple opportunities have received limited attention in the literature. One exception was a direct comparison of the intent of the prescribing physicians with the patients' interpretations of the prescription ${ }^{[9]}$. In other words, the researchers asked the prescribing physicians how they had intended for the patient to take the drug and asked the patients how they were actually taking them. The findings revealed discrepancies for 44 of the 115 (38\%) prescription instructions. Recent research further identified significant variability in pharmacists' interpretation and subsequent conveyance of physicians' prescription instructions ${ }^{[10]}$.

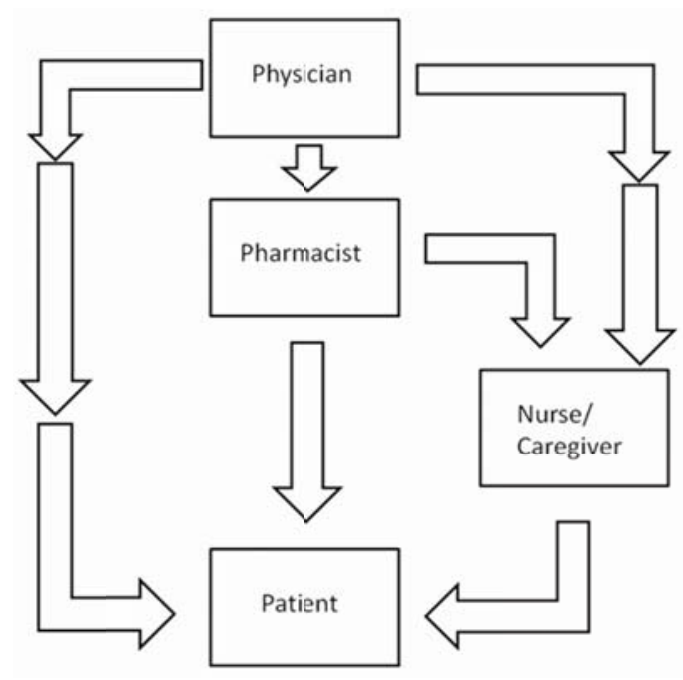

Figure 1. Conceptualization of the information flow of prescription instructions and the resulting multiple opportunities for misinterpretation 
The present studies were designed to assess agreement among interpretations within and between various stages during the flow of information. In prior studies reporting agreement in interpretation between patients and the prescribing physiccians ${ }^{[9]}$, the physicians were the prescribing agents and were aware their own intentions. The purpose of the present experiment was to investigate the agreement of common prescription instructions by those providing the instructions physicians, as compared to a group of young healthcare consumers. In addition, nurses are the professionals most often charged with the task of providing discharge instructions to patients prior to release from various healthcare facilities. Consequently, nurses comprise a vital link in the information flow between a physician and a patient. Thus, practicing RNs were later asked to complete the questionnaire for comparison.

Our goal was to examine how a broad range of common prescription instructions were interpreted by these three groups and to further determine which prescriptions were most problematic and which were most consistently understood. More specifically, our objective was to identify discrepancies in interpretation of prescription instructions between healthcare consumers, nurses, and physicians.

\section{Methods}

The present study was a cross-sectional comparison of interpretations of common prescription instructions by young healthcare consumers, physicians, and nurses. The interpretation questionnaire was open-ended and asked participants when they would take a drug based on a given prescription instruction (e.g., "If your prescription reads 'Take one tablet every twelve hours,' at what times of the day would you take the drug?"). In order to determine any possible influence of the frequency of food consumption on the interpretation of the prescription instruction, "take one capsule with meals," the undergraduates were also asked to indicate the number of meals they eat per day. Physicians and nurses completed a questionnaire with identical prescription instructions but modified to measure the physicians' intent regarding a given prescription instruction (e.g., "If you prescribe 'Take one tablet every twelve hours,' at what times of the day would you prefer your patient to take the drug?") or the nurses' interpretation of the physicians intent (e.g., "If a physician prescribes 'Take one tablet every twelve hours,' at what times of the day would you prefer your patient to take the drug?"). For the specific prescription instructions refer to Table 1.

\subsection{Participants}

Convenience sampling was utilized to create three groups for comparison (undergraduates, nurses, and physicians). Approval to administer the questionnaires was obtained from the Institutional Review Boards of the respective institutions from which participants were recruited. Seventy-four undergraduate participants were recruited from lower-level psychology courses at a large southern university to comprise the group of young healthcare consumers. The undergraduate participants signed informed consents and completed the interpretation questionnaire in groups of one to six participants. The undergraduates earned extra credit or fulfilled a partial course requirement. Upon completion, the questionnaires were collected and the participants were debriefed.

Physicians were recruited from a mid-size hospital in the southern United States. Hospital administrators announced the opportunity to complete the survey on a voluntary basis. The surveys were made available via the physicians lounge and self-submitted anonymously via a collection box. Thirty-six physicians ultimately completed the survey. Practicing RNs were recruited from additional clinical settings in a similar manner. Thirty-four practicing RNs ultimately completed the survey. Neither physicians nor RNs were compensated for their participation.

\subsection{Data-analysis}

The survey responses were entered into Microsoft Excel spreadsheets. The percentages for each response provided per group were then noted (e.g., $45 \%$ of undergraduates stated, "breakfast and bedtime"). When appropriate, frequencies or time-intervals were calculated based on the provided interpretation (e.g., $62 \%$ of undergraduates interpreted 3 dosages per 
day). Once these percentages were calculated, a Chi-squared analysis was calculated by hand. Because "don't prescribe this way" is not a response applicable to nurses or undergraduates, two prescription instructions were excluded from the Chi-squared analysis: a) "take one tablet every $12 \mathrm{hrs,"} \mathrm{and} \mathrm{b)} \mathrm{take} \mathrm{one} \mathrm{capsule} \mathrm{on} \mathrm{an} \mathrm{empty} \mathrm{stomach.}$

\section{Results}

Table 1 displays the interpretation/intent of each of the prescription instructions by physicians, nurses, and undergraduates. It is evident in this table that differences in interpretations occurred both within our groups of physicians, nurses, and undergraduates and between these groups. This impression was confirmed by a Chi-squared analysis $\chi^{2}(30)=$ $134.9, p<.001)$.

In several cases, the different interpretations would have little pharmaceutical import. 'Take twice daily' was understood by all but $12 \%$ of undergraduates to imply morning and evening doses. Yet $11 \%$ chose a schedule where the doses were approximately 4-5 hours apart and 1\% reported taking both tablets at once. "Take one capsule with meals" was similarly problematic, with $34 \%$ of undergraduates reporting less than 3 dosages/day. No prescription instruction was uniformly interpreted by physicians, nurses, or undergraduates.

Table 1. Interpretation or Intent (Physicians Only) for Common Prescription Instructions

\begin{tabular}{|c|c|c|c|c|}
\hline \multirow{2}{*}{ Instruction } & \multirow{2}{*}{ Interpretation/Intent } & \multicolumn{3}{|c|}{ Percentage Response from Each Participant Group } \\
\hline & & Physicians & Nurses & Undergraduates \\
\hline 'Take once a day' & breakfast & $72 \%$ & $88 \%$ & $50 \%$ \\
\hline \multirow{6}{*}{ 'Take twice daily' } & breakfast \& dinner & $58 \%$ & $15 \%$ & $32 \%$ \\
\hline & breakfast \& 8pm & $17 \%$ & $38 \%$ & - \\
\hline & breakfast $\&$ bedtime & $17 \%$ & $24 \%$ & $45 \%$ \\
\hline & lunch \& dinner & - & - & $8 \%$ \\
\hline & breakfast \& lunch & - & $3 \%$ & $3 \%$ \\
\hline & two pills at once & - & - & $1 \%$ \\
\hline \multirow{2}{*}{$\begin{array}{l}\text { 'Take one tablet } \\
\text { every twelve hours' }\end{array}$} & $12 \mathrm{hr}$ intervals & $97 \%$ & $100 \%$ & $97 \%$ \\
\hline & don't prescribe this way & $3 \%$ & - & - \\
\hline \multirow{4}{*}{$\begin{array}{l}\text { 'Take one capsule } \\
\text { with meals' }\end{array}$} & 3 dosages per day & $72 \%$ & $59 \%$ & $62 \%$ \\
\hline & 2 dosages per day & - & $3 \%$ & $12 \%$ \\
\hline & 1 dosage per day & $6 \%$ & $32 \%$ & $22 \%$ \\
\hline & When they eat & $20 \%$ & $6 \%$ & - \\
\hline \multirow{2}{*}{$\begin{array}{l}\text { 'Take one capsule on } \\
\text { an empty stomach' }\end{array}$} & before breakfast & $69 \%$ & $71 \%$ & $-{ }^{a}$ \\
\hline & don't prescribe this way & $8 \%$ & - & - \\
\hline \multirow{4}{*}{$\begin{array}{l}\text { 'Take one tablet four } \\
\text { times a day' }\end{array}$} & 4-hour intervals ${ }^{b}$ & $8 \%$ & $56 \%$ & $20 \%$ \\
\hline & 5-hour intervals ${ }^{b}$ & $83 \%$ & $15 \%$ & $73 \%$ \\
\hline & 6-hour intervals & - & $15 \%$ & - \\
\hline & $\begin{array}{l}\text { Less than 4-hour } \\
\text { intervals }{ }^{c}\end{array}$ & - & $12 \%$ & $5 \%$ \\
\hline
\end{tabular}

Note. ${ }^{\mathrm{a}}$ The undergraduate response were quite varied but seemed to adhere to the intent of the prescription instruction. ${ }^{\mathrm{b}}$ typical waking hours only.

${ }^{\mathrm{c}}$ Interpretations were reported with as few as two hours separating each dose ('8am, 10am, noon, 2pm').

\section{Discussion}

Previous studies have indicated a discrepancy in the interpretation of intended dosage between actual patients and the prescribing physician for prescription instructions labeled "as directed" ${ }^{[9]}$ and wide variability in the interpretation of 
common prescription instructions ${ }^{[5]}$. The present study was intended to empirically measure the variability of both physicians' and nurses' interpretations of common prescription instructions. The results indicated considerable variability in the interpretation of prescription instructions both within each group of physicians, nurses, and undergraduates as well as discrepancies between these groups.

Comparing the (relatively) unambiguous prescriptions with the more ambiguous ones, we find prescriptions that mention explicit temporal schedules ("One tablet every 12 hours") are more consistently interpreted than are frequency-based prescriptions ("Twice daily"), which are more consistently interpreted than event-based prescriptions ("One capsule with meals").

Event-based prescriptions are especially problematic because they contain an inherent ambiguity. $72 \%$ of physicians interpreted "one capsule with meals" as being equivalent to 3 dosages per day, spaced out at equal intervals and perhaps buffered by the presence of food in the stomach. Yet the medication might be tied with meals because of a gastrointestinal action and so only needs to be taken when a meal is consumed. In that case, patients who only eat two meals per day should receive only two dosages per day.

Of the $41 \%$ of our undergraduates who reported eating only two meals per day, three-fourths indicated they would take three dosages per day, matching the apparent physician's intent. But one-fourth indicated they would take only two dosages per day. One-fourth of physicians seemed insensitive to this ambiguity and explained their intent with phrases such as "with food" or "when they eat." In such cases, clarifications such as "when they eat" do not resolve the ambiguity of determining whether the pill should be taken one or more times a day.

Standardization is commonly proposed as a solution to reducing errors and increasing adherence. However, standardization is less straightforward than it may appear. An investigation of computerized prescription instructions over a three month period revealed that $15 \%$ of the prescription instructions received additional words or changes in wording ${ }^{[1]}$. Moreover, researchers recently identified significant variability in pharmacists' interpretations of physicians' prescription instructions ${ }^{[10]}$. Thus, awareness that prescription instructions can be interpreted with such variability is a first step to addressing the problem. More critically, standardization does not address the problem identified in the present study.

It should be noted that are several potential limitations of the present study, including the small sample size. Another potential limitation of the present study is that the instructions were independent of any actual drugs. This could have resulted in an increase in the variability observed among the healthcare providers. Nonetheless, specific drugs are often prescribed in differing amounts depending on the patients' needs, underscoring the need to address the issue of varied interpretations. Future studies can explore variability of prescription instructions when specific drugs are used in conjunction with the instructions. A related possible limitation is the lack of interaction among the groups. In many real-life healthcare situations, there are opportunities for some degree of provider/patient interactions. In the present investigation, the groups were interpreting the prescription instructions independent of such interactions. Thus, while the present study revealed differences in interpretations for common prescription instructions, future studies can explore the potential mediating effect of provider/patient interaction on interpretations.

\section{I mplications for nursing practice}

The results of the present study have broad implications for practicing nurses and educators alike. Nurses maintain a pivotal role in the education of patients regarding their therapeutic regimens. As such, nurses require education in educational principles and in the psychology of learning. The results of the current study reflect the presence of broad misperceptions regarding common medication instructions on the part of both patients and nurses. It is essential for nurses to integrate patient educational approaches that do not fail to ensure, rather than assume, that patients recognize the dosing pattern of their medications. In truth, the results reflect the likelihood that even the simplest instructions are likely to be misinterpreted, resulted in an increased likelihood of treatment related injury. 


\section{Conclusion}

The present study explored interpretations (or intent) of prescription instructions of physicians and nurses, as well as young healthcare consumers. As the prescribing agents, physicians are the first step in the flow of information required for patients to obtain their prescriptions. Thus, these combined reports indicate the enormous potential for error introduced not only at the level of patient misinterpretation but from physician to pharmacist to patient. Moreover, the introduction of a nurse or other provider introduces an additional opportunity for misinterpretation. In conclusion, given the financial and human costs of misinterpreting prescription instructions, an awareness of potential sources of misinterpretation at each stage of the flow of information is vital.

\section{References}

[1] Kohn KT, Corrigan JM, Donaldson MS, editors. To err is human: building a safer health system. Washington, DC: National Academy Press; 1999.

[2] Bates DW, Cullen DJ, Laird N, et al. Incidence of adverse drug events. JAMA. 1995; 274: 29-34. PMid:7791255 http://dx.doi.org/10.1001/jama.274.1.29

[3] Phillips DP, Christenfield N, Glynn LM. Increase in US medication-error deaths between 1983 and 1993. Lancet. 1998; 351 : 643-644. http://dx.doi.org/10.1016/S0140-6736(98)24009-8

[4] Sabate E. Adherence to long-term therapies: evidence for action. Geneva: World Health Organization. 2003. Available from: http://www.who.int/chp/knowledge/publications/adherence_full_report.pdf

[5] Mazzullo JM, Lasagna L, Griner PF. Variations in interpretation of prescription instructions. JAMA. 1974; 227 : $929-931$. PMid:4405864 http://dx.doi.org/10.1001/jama.1974.03230210039009

[6] Malahy B. The effect of instruction and labeling on the number of medication errors made by patients at home. Am Journal Health-Syst Pharm. 1966; 23: 283-292.

[7] Davis TC, Wolf MS, Bass PF, et al. Low literacy impairs comprehension of prescription drug warning labels. J Gen Intern Med. 2006; 21: 847-51. PMid:16881945 http://dx.doi.org/10.1111/j.1525-1497.2006.00529.x

[8] Wolf MS, Davis TC, ShrankW, et al. To err is human: patient misinterpretations of prescription drug label instructions. Patient Educ Couns. 2007; 67: 293-300. PMid:17587533 http://dx.doi.org/10.1016/j.pec.2007.03.024

[9] Powell JR, Cali TJ, Linkewich JA. Inadequately written prescriptions; “As directed” prescriptions analyzed. JAMA. 1973; 226: 999-1000. PMid:4800343 http://dx.doi.org/10.1001/jama.1973.03230080139027

[10] Wolf MS, Shekelle P, Choudhry NK, et al. Variability in pharmacy interpretations of physician prescriptions. Med Care. 2009; 47: 370-373. PMid:19194338 http://dx.doi.org/10.1097/MLR.0b013e31818af91a

[11] Reti RS. Pharmacy prescription additions. NZFP. 2001; 28: 411-414. 